\title{
$\alpha$-taxilin overexpression correlates with proliferation activity but not with prognosis of colorectal cancer
}

\author{
AKIRA KANAMORI $^{1}$, YASUO IMAI $^{2}$, KEISUKE IHARA $^{3}$, HITOSHI NAGATA $^{4}$, \\ MASAKAZU NAKANO ${ }^{1}$, KEIICHI TOMINAGA ${ }^{1}$, HIROAKI SHIMIZU ${ }^{5}$, TOMIHIKO MAKIYAMA ${ }^{5}$, \\ HAJIME KURODA ${ }^{2}$, HIROMICHI SHIRATAKI ${ }^{5}$ and HIDEYUKI HIRAISHI ${ }^{1}$ \\ Departments of ${ }^{1}$ Gastroenterology, ${ }^{2}$ Diagnostic Pathology, ${ }^{3}$ Surgical Oncology, ${ }^{4}$ Gastroenterological Surgery, and \\ ${ }^{5}$ Molecular and Cell Biology, School of Medicine, Dokkyo Medical University, Mibu, Tochigi 321-0293, Japan
}

Received December 19, 2016; Accepted May 23, 2017

DOI: $10.3892 / 01.2017 .6309$

\begin{abstract}
. $\alpha$-taxilin is a binding partner of syntaxins, which are the central coordinators of membrane traffic. Expression of $\alpha$-taxilin has been implicated in the development of human glioblastoma, hepatocellular carcinoma and renal cell carcinoma. In the present study, the clinical significance of $\alpha$-taxilin expression in colorectal cancer (CRC) was investigated. A total of 20 cases of colorectal intramucosal adenocarcinoma (IMA) with adenoma were analyzed using immunohistochemical analysis. The results demonstrated that $\alpha$-taxilin expression was significantly associated with Ki-67 indices in adenoma and IMA. The patients expressed equally high levels of $\alpha$-taxilin in the upper third of the intramucosal glands. These results suggest that $\alpha$-taxilin expression is significantly associated with the proliferative activity of CRC, but that its overexpression alone is not a biomarker of malignancy. Next, $\alpha$-taxilin expression was investigated in 57 advanced CRCs and its association with prognosis was determined. Well-differentiated and/or moderately differentiated adenocarcinomas in the left-sided colon with anatomic stage II and/or III were analyzed. $\alpha$-taxilin expression levels were high on the surface of nearly all tumors, but variable at the deep advancing edge. $\alpha$-taxilin levels at the advancing edge were not significantly associated with local invasiveness or prognosis. In conclusion, $\alpha$-taxilin is a cell proliferation marker in colorectal epithelial neoplasms but cannot be a marker of malignancy or prognosis of CRCs.
\end{abstract}

Correspondence to: Professor Yasuo Imai, Department of Diagnostic Pathology, School of Medicine, Dokkyo Medical University, 880 Kitakobayashi, Shimotsuga, Mibu, Tochigi 321-0293, Japan

E-mail: ya-imai@dokkyomed.ac.jp

Key words: $\alpha$-taxilin, colorectal cancer, proliferation, Ki-67, prognosis

\section{Introduction}

Colorectal cancer (CRC) is the third most common cancer and the fourth leading cause of cancer-related death worldwide $(1,2)$. In 2012, 1,360,600 new cases were diagnosed and 693,900 deaths were attributed to CRC (2). The carcinogenetic mechanism of CRC has been extensively investigated and intracellular signaling pathways related to cell survival, cell fate, and genome maintenance have been implicated (3).

Membrane traffic is a fundamental intracellular transport system in eukaryotic cells, and recent studies have revealed that molecules involved in membrane traffic play an important role in the initiation and progression of several types of tumor (4-6). Soluble $N$-ethylmaleimide-sensitive factor attachment protein receptors (SNAREs) located on intracellular vesicles and their target membranes are the central coordinators of membrane traffic (7), and syntaxin family proteins are the main components of SNARE complexes. Taxilin was identified as a novel binding partner of syntaxins (8). The taxilin family consists of at least three members: $\alpha-, \beta-$, and $\gamma$-taxilins. $\alpha$-taxilin binds to free syntaxins that are not part of a SNARE complex (9), suggesting that $\alpha$-taxilin may act as a regulator of vesicular transport by affecting the assembly of SNARE complexes. In the physiological setting $\alpha$-taxilin was proposed to be involved in $\mathrm{Ca}^{2+}$-dependent exocytosis in neuroendocrine cells (8), and it was recently reported that $\alpha$-taxilin is normally expressed in gastrointestinal epithelial cells (10). $\alpha$-taxilin is also expressed in stromal cells, such as mouse fibroblast NIH3T3 (10) and human fibrosarcoma HT1080 (11) cell lines. Overexpression of $\alpha$-taxilin mRNA has been reported in human glioblastoma compared with normal tissues of the central nervous system (12). $\alpha$-taxilin expression has also been found to be associated with proliferative activity and dedifferentiation of hepatocellular carcinoma (HCC) (13) and local invasiveness and poor prognosis of renal cell carcinoma (RCC) (14).

In the present study, we analyzed $\alpha$-taxilin expression in human colorectal tumors and explored the associations between $\alpha$-taxilin overexpression and prognosis of CRC. This is the first study to investigate the clinical significance of $\alpha$-taxilin in human CRC. 


\section{Materials and methods}

Tumor samples, pathological diagnosis, and staging. CRCs that were endoscopically or surgically resected at the Dokkyo Medical University Hospital (DMUH) and the International University of Health and Welfare, Shioya Hospital (IUHWSH) were analyzed. Clinicopathologic classification was based on the World Health Organization classification of colorectal tumors and stage grouping was according to tumor, node, metastases (TNM) staging of the American Joint Committee on Cancer (AJCC) Colon and Rectum Cancer Staging $(15,16)$.

Colorectal intramucosal adenocarcinomas (IMAs; pTis defined by AJCC) with adenoma components were selected from all CRCs resected at IUHWSH from 2013 to 2015 and at DMUH from 2012 to 2014. A total of 20 IMAs, pathologically diagnosed as well-differentiated and/or moderately differentiated adenocarcinoma, were analyzed. For pathological analysis, intramucosal glands were divided into three anatomical components: Upper third (UT), middle third (MT), and lower third (LT) (Fig. 1A). Next, among all CRCs surgically resected at DMUH and IUHWSH from 2009 to 2011, histologically proven well-differentiated and/or moderately differentiated adenocarcinomas in the left-sided colon with anatomic stage II and/or III were selected. All CRCs included in this study were diagnosed clinically and pathologically as primary tumors and confirmed to be advanced cancers that invaded the muscularis propria or more (pT2 to 4). We aimed to study the pure effect of $\alpha$-taxilin expression on prognosis by normalizing other possible prognostic factors, such as tumor histology, differentiation grade, location, and initial anatomic stage (17). Patients with complete medical records were included in the survival analyses, but individuals with invasive cancers originating from other sites were excluded. As a result, a total of 57 cases were subjected to prognostic analyses. This study protocol was approved by the ethical review boards of the participating hospitals (IUHWSH, FK-94; DMUH, 26067).

Immunohistochemistry. Tumor specimens were fixed in $10 \%$ neutral-buffered formalin for $48 \mathrm{~h}$, embedded in paraffin, and then cut into $4-\mu \mathrm{m}$ sections. Antigen retrieval was performed in $10 \mathrm{mM}$ citrate buffer ( $\mathrm{pH}$ 6.0) using microwave irradiation $(400 \mathrm{~W})$ at $95^{\circ} \mathrm{C}$ for $40 \mathrm{~min}$. After quenching endogenous peroxidase activity, sections were incubated with primary antibody detecting $\alpha$-taxilin (1:1,000) or Ki-67 (1:50, clone: Mib-1; Dako, Glostrup, Denmark) for $60 \mathrm{~min}$ at room temperature. Characterization of the anti- $\alpha$-taxilin antibody was described in previous studies $(10,18)$. Intensity of $\alpha$-taxilin staining was classified into four categories: Level 3, comparable to that of proliferating cells in the lower crypt, follicular dendritic cells, and immunoblasts: Level 2, comparable to that of ganglion cells and follicular B-lymphocytes: Level 1, between level 0 and 2: Level 0, no staining (Fig. 1B and C). $\alpha$-taxilin expression of levels 2 and 3 was regarded as overexpression. Ki-67 indices were calculated as the percentage of Ki-67-positive cells among 500 to 1,000 cells in the areas with the strongest nuclear labeling.

Statistics. Comparisons between two sets of data were performed by non-paired/paired two-tailed Student's t-test. Correlation between $\alpha$-taxilin expression levels and Ki-67
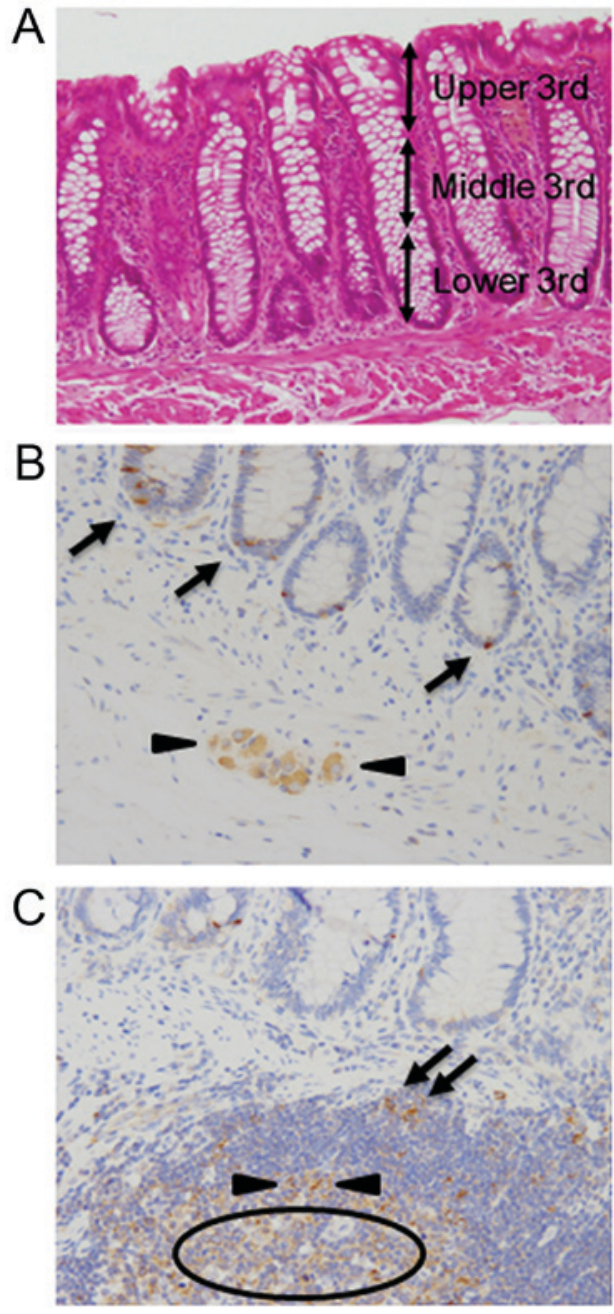

Figure 1. (A) Three anatomical components of normal colonic crypts: Upper third, middle third, and lower third. (B) $\alpha$-Taxilin expression in the colonic crypts and ganglion cells. Level 3, comparable to expression of proliferating cells in the lower crypt (arrows); level 2, comparable to expression of ganglion cells (arrowheads). (C) $\alpha$-Taxilin expression in the lymph follicles. Level 3, comparable to expression of follicular dendritic cells (arrowheads) and immunoblasts (arrows); level 2, comparable to expression of follicular B lymphocytes (in the circle).

indices were analyzed by Spearman's rank correlation analysis. Specific parameters between two patient cohorts were compared using the $\chi^{2}$ test with/without Yates' correction or by Fisher's exact test. Age was compared using the Mann-Whitney U test, and survival curves were analyzed using the Kaplan-Meier method and log-rank tests. $\mathrm{P}<0.05$ was considered to indicate a statistically significant difference. Statistical analyses were performed using IBM SPSS Statistics 23 (IBM SPSS, Armonk, NY, USA).

\section{Results}

$\alpha$-taxilin expression in IMA and adenoma. A total of 20 cases of IMA with adenoma were analyzed (Fig. 2A). The proliferative zone was in the LT in the normal colonic crypt, whereas it shifted to UT in adenoma and spread from the UT downward in IMA (Fig. 2B). $\alpha$-taxilin expression paralleled Ki-67 expression (Fig. 2B and C). In the normal mucosa, expression of both $\alpha$-taxilin and Ki-67 was strongest in LT followed by 


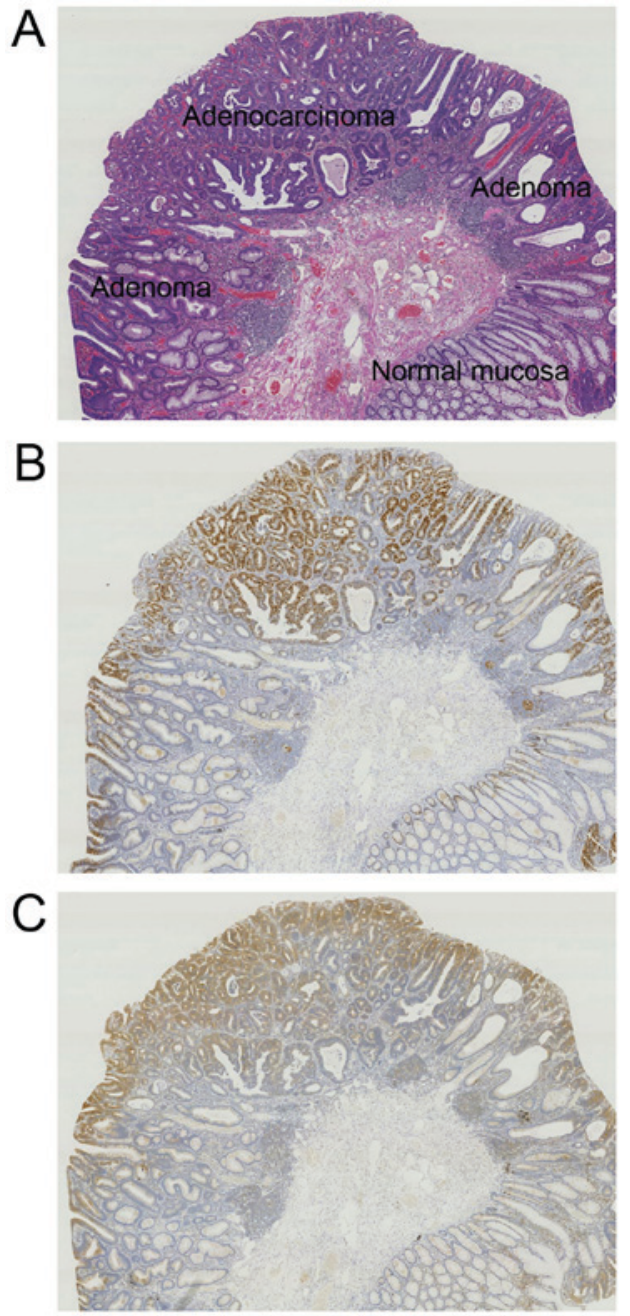

Figure 2. (A) Representative histological structure of IMA with adenoma (H\&E x2). (B) Ki-67 expression status (x2). (C) $\alpha$-Taxilin expression status (x2). IMA, intramucosal adenocarcinoma; H\&E, hematoxylin and eosin.

MT, and weakest in UT of the crypt (Fig. 3A). In contrast, for glands in adenoma and IMA, expression of both $\alpha$-taxilin and Ki-67 was strongest in UT followed by MT, and weakest in LT (Fig. 3A). $\alpha$-taxilin levels were similarly high in UT of glands of adenoma and IMA $(2.813 \pm 0.527$ and $2.875 \pm 0.484$, respectively), although Ki-67 indices were significantly higher in IMA than in adenoma $(88.46 \pm 9.343$ and $63.35 \pm 17.2$, respectively; $\mathrm{P}<0.01$ ) (Fig. 3A).

Coordinate points consisting of $\alpha$-taxilin level in the $\mathrm{x}$-axis and $\mathrm{Ki}-67$ index in the y-axis in respective anatomical components (LT, MT and UT) of intramucosal neoplastic glands were plotted in a scattergram (Fig. 3B). $\alpha$-taxilin level and Ki-67 index showed a significant positive correlation in both adenoma and IMA.

$\alpha$-taxilin expression in CRC and prognosis. We next investigated $\alpha$-taxilin expression in histologically proven well-differentiated and/or moderately differentiated adenocarcinoma in the left-sided colon with anatomic stage II and/or III and examined its association with local invasiveness and prognosis. $\alpha$-taxilin was strongly expressed at the cancer surface of nearly all CRCs: Level 1 in 6 cases, level 2 in 15 cases, level 3 in 35 cases, not determined in one case (Fig. 4A and C).
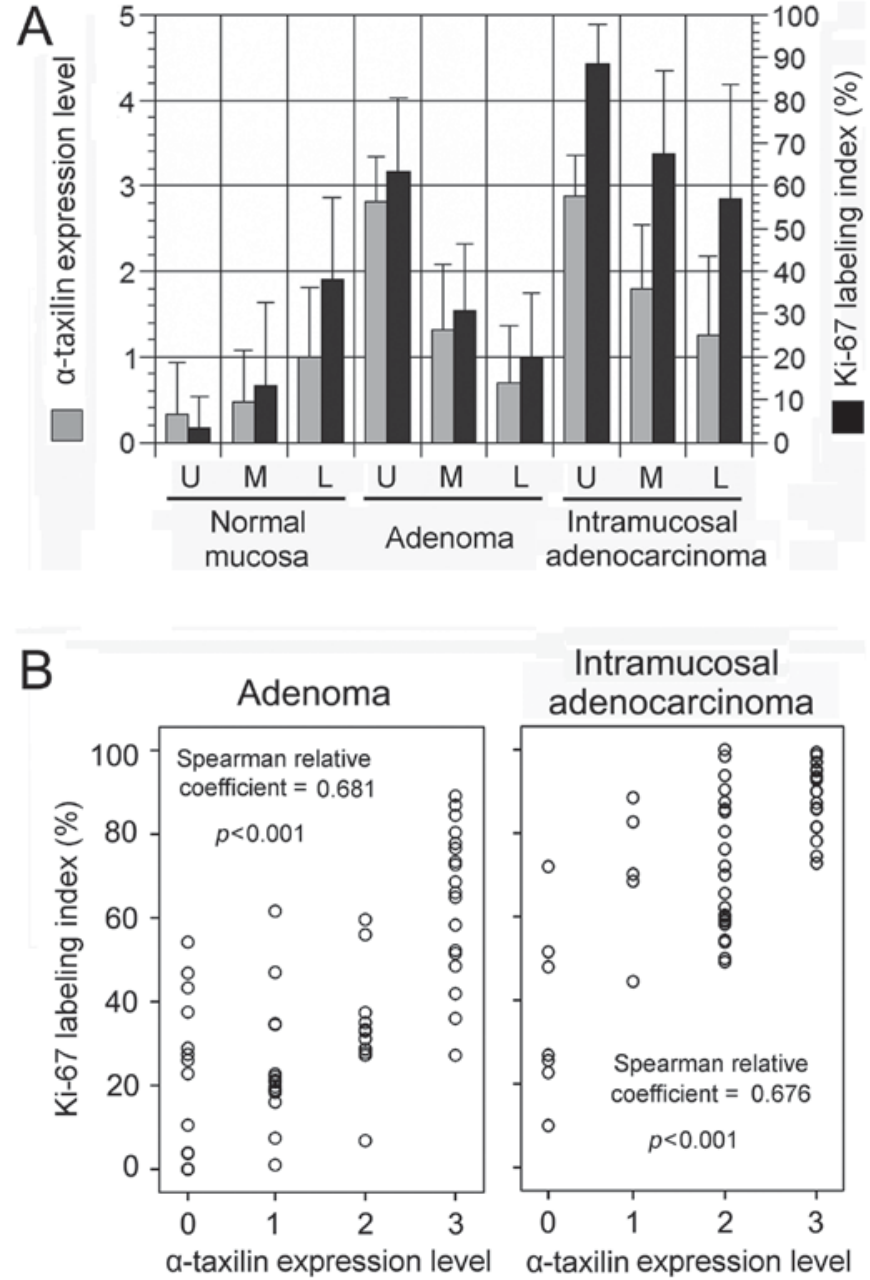

Figure 3. (A) $\alpha$-Taxilin expression levels and Ki-67 labeling indices in the respective anatomical units (lower, middle and upper thirds) of the normal crypt, adenoma, and IMA. (B) Scattergrams displaying correlations between $\alpha$-taxilin expression level and $\mathrm{Ki}-67$ labeling index in three anatomical units of adenoma and IMA. U, upper third; M, middle third; L, lower third; IMA, intramucosal adenocarcinoma.

In contrast, $\alpha$-taxilin expression levels at the cancer deep advancing edge in the colorectal wall were more variable: Level 0 in 4 cases, level 1 in 12 cases, level 2 in 25 cases, and level 3 in 16 cases (Fig. 4B and D). Sixteen of 57 cases (28.1\%) showed low expression of $\alpha$-taxilin. On average, there was a significant difference in $\alpha$-taxilin expression levels between the surface area and the advancing edge $(2.52 \pm 0.09$ and $1.91 \pm 0.12$, respectively; $\mathrm{P}<0.01)$ in the present $\mathrm{CRC}$ cohorts.

Malignant tumor is characterized by destructive downward growth and metastasis. We therefore investigated associations of $\alpha$-taxilin expression in cancer cells at the advancing edge with local invasiveness and prognosis. $\alpha$-taxilin overexpression (levels 2 and 3) was not associated with any of the clinical parameters tested, including depth of tumor (pT), venous invasion, lymphatic permeation, nodal metastasis, and clinical stage (Table I). In the survival analyses, CRCs with $\alpha$-taxilin overexpression showed a trend for worse 5-year recurrence-free survival than those without $\alpha$-taxilin overexpression, but the difference did not reach statistical significance (Fig. 5A). Advanced CRCs with and without $\alpha$-taxilin overexpression demonstrated similar 5-year overall survival (Fig. 5B). These 
Table I. Association between $\alpha$-taxilin overexpression in cancer cells at the deep advancing edge and clinicopathologic characteristics of advanced CRC.

\begin{tabular}{|c|c|c|c|}
\hline \multirow[b]{2}{*}{ Characteristics } & \multicolumn{2}{|c|}{$\alpha$-Taxilin overexpression } & \multirow[b]{2}{*}{ P-value } \\
\hline & Yes $(n=41)$ & No $(n=16)$ & \\
\hline \multicolumn{4}{|l|}{ Age } \\
\hline Range (mean) & $42-83(67)$ & $58-81(69.5)$ & 0.505 \\
\hline \multicolumn{4}{|l|}{ Gender } \\
\hline Male & 23 & 8 & 0.771 \\
\hline Female & 18 & 8 & \\
\hline \multicolumn{4}{|l|}{ pT4 } \\
\hline Yes & 8 & 4 & 0.450 \\
\hline No & 33 & 12 & \\
\hline \multicolumn{4}{|l|}{ Venous invasion } \\
\hline Yes & 28 & 11 & 1.000 \\
\hline No & 13 & 5 & \\
\hline \multicolumn{4}{|c|}{ Lymphatic permeation } \\
\hline Yes & 32 & 13 & 1.000 \\
\hline No & 9 & 3 & \\
\hline \multicolumn{4}{|l|}{ Nodal metastasis } \\
\hline Yes & 19 & 8 & 1.000 \\
\hline No & 22 & 8 & \\
\hline \multicolumn{4}{|l|}{ Clinical stage } \\
\hline II & 22 & 8 & 1.000 \\
\hline III & 19 & 8 & \\
\hline
\end{tabular}

CRC, colorectal cancer; pT4, tumor penetrating to the surface of the visceral peritoneum, directly invading or adherent to other organs or structures.

results suggest that the $\alpha$-taxilin expression level may not affect prognosis of CRCs.

\section{Discussion}

It has been reported that $\alpha$-taxilin is related to cell proliferation of neuroepithelial cells and malignancies of epithelial origin, such as HCC and RCC $(8,13,14)$. Recently, Horii et al reported $\alpha$-taxilin expression status in the normal gastrointestinal tract in mice (10). They demonstrated that $\alpha$-taxilin was expressed in the majority of the gastrointestinal tract and prominently present in the cytoplasm of epithelial cells expressing Ki-67 in C57BL/6 mice. Although some epithelial cells expressed $\alpha$-taxilin without Ki-67 expression, the majority of $\alpha$-taxilin-positive/Ki-67-negative epithelial cells were observed in the vicinity of $\alpha$-taxilin/Ki-67 double-positive cells, and the authors reasoned that $\alpha$-taxilin remained in these cells following division and subsequent loss of Ki-67 expression. They also reported that treatment with dibenzazepine, a $\gamma$-secretase inhibitor that inhibits cell proliferation in the stomach, resulted in a decrease in the number of $\mathrm{Ki}$-67-positive cells and $\alpha$-taxilin-positive cells in the lower part of the gastric glands, suggesting that expression of

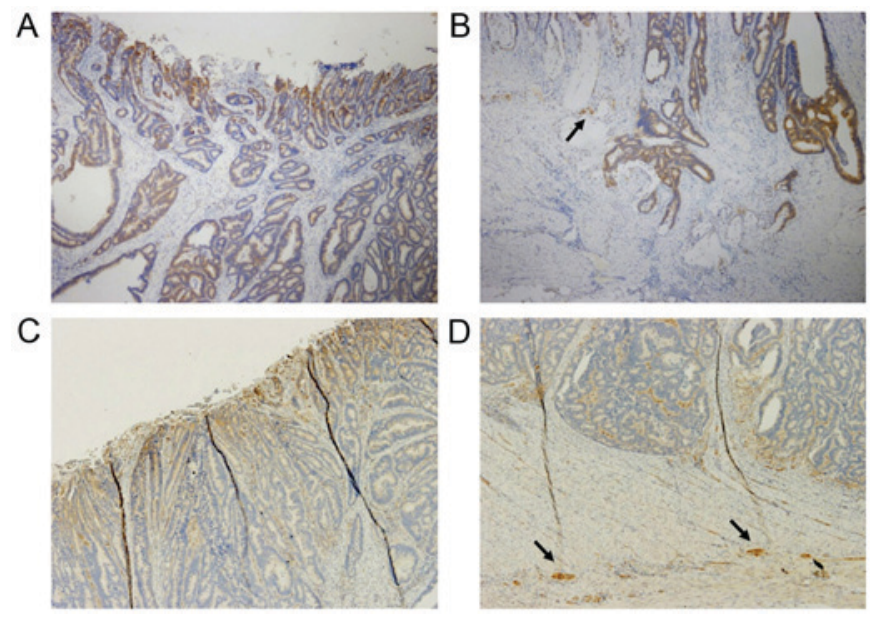

Figure 4. Representative $\alpha$-taxilin expression status at the surface and at the deep advancing edge of advanced CRC (x4). Level 3 staining in the (A) surface area and at the (B) advancing edge in the sigmoid colon cancer of an 80-year-old male patient. Arrows indicate ganglion cells. Level 3 staining at the (C) surface area and level 1 staining at the (D) advancing edge in rectal cancer of a 78-year-old female patient. Arrows indicate ganglion cells. CRC, colorectal cancer.
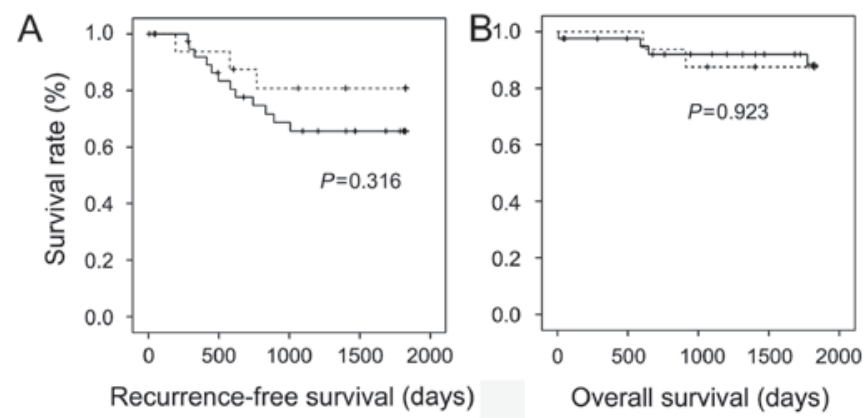

Figure 5. Survival analyses of histologically proven well-differentiated and/or moderately differentiated adenocarcinoma in the left-sided colon with anatomic stage II and/or III. (A) 5-year recurrence-free survival in the presence $(n=41$; solid line) or absence $(n=16$; dotted line) of $\alpha$-taxilin overexpression at the deep advancing edge. (B) 5-year overall survival in the presence $(n=41$; solid line) or absence $(n=16$; dotted line) of $\alpha$-taxilin overexpression at the deep advancing edge.

$\alpha$-taxilin was dependent on cell proliferation. In the present study, we investigated $\alpha$-taxilin expression status in human colorectal tumors for the first time. Assuming that $\alpha$-taxilin might be a marker of CRC, we first studied its expression focusing on the pathological sequence of colorectal carcinogenesis. In normal mucosa, the proliferation zone of the colonic crypt is located in the lower third of the crypt, where stem cells are present. However, the proliferation zone represented by high Ki-67 indices shifted to the upper third of the crypt in adenoma and was found to extend downward from the UT in IMA. These observations corresponded well with one of the two models explaining colon carcinogenesis: The top-down model. This model proposes that transformation is initiated in a fully differentiated cell in which adenomatous polyposis coli is highly expressed and $\beta$-catenin is downregulated (19). The fully differentiated villus cell proliferates and replaces the normal mucosa from the top down. In contrast, the bottom-up model proposes that transformation is initiated 
in a stem cell at the base of the crypt, which proliferates and replaces the normal mucosa with transformed cells from the bottom up. In the present study, $\alpha$-taxilin levels were significantly associated with proliferation activity in both adenoma and IMA. We observed that $\alpha$-taxilin was similarly upregulated in the upper third of the neoplastic glands in both adenoma and IMA. These data suggested that $\alpha$-taxilin expression status might be similar between adenoma and adenocarcinoma, and that high $\alpha$-taxilin levels could not be a marker of malignancy in the large intestine.

We next investigated $\alpha$-taxilin expression in histologically proven well-differentiated and/or moderately differentiated adenocarcinoma in the left-sided colon with anatomic stage II and/or III in order to investigate its prognostic significance in CRC. Since $\alpha$-taxilin expression levels were similarly high in the surface area of adenoma and adenocarcinoma, we reasoned that $\alpha$-taxilin expression on the surface could not be a marker of malignancy or prognosis in CRCs. However, the malignant potential of cancer would be determined by proliferation activity, destructive downward growth, and metastasis, suggesting that malignant potential may be determined by the invasiveness and proliferation activity of cancer cells at the deep advancing edge. We therefore investigated associations of $\alpha$-taxilin expression at the advancing edge with local invasiveness (pT and vessel invasion) and prognosis; however, $\alpha$-taxilin expression levels were not associated with local invasiveness and also did not affect 5-year recurrence-free survival or overall survival of patients with advanced CRCs.

Previously, Ohtomo et al reported that $\alpha$-taxilin upregulation was significantly associated with proliferative activity, less-differentiated histological grade, positivity of vascular invasion, and/or intrahepatic metastasis of HCC (13). In addition, Mashidori et al reported that $\alpha$-taxilin upregulation was significantly associated with depth of tumor (pT), vessel invasion, and unfavorable overall and disease-free survival of patients with RCC (14). In the present study, $\alpha$-taxilin expression levels were associated with proliferation activity but its overexpression did not significantly affect prognosis of patients with CRC. There are some possible explanations for this discrepancy. First, it is well known that a majority of CRCs develop via the adenoma-carcinoma sequence. Because $\alpha$-taxilin levels in adenoma cells are already as high as those in adenocarcinoma cells, upregulation of $\alpha$-taxilin alone cannot be a marker of malignant potential of colorectal neoplasms. In contrast, except for a few cases, no such common precancerous lesion has been reported for HCC and RCC. The majority of both tumors may develop de novo, and therefore $\alpha$-taxilin upregulation could be a marker of malignancy. Second, we selected a relatively homogenous group of CRCs to investigate only the effect of $\alpha$-taxilin expression on prognosis. We enrolled only cases of histologically proven differentiated adenocarcinoma in the left-sided colon with anatomic stage II and/or III that underwent surgical resection. However, this selection might have resulted in selection bias because $\alpha$-taxilin levels might have affected pT and nodal/distant metastasis. Analysis of all consecutive CRC cases in our regional cohort during the study period might have resulted in a different conclusion. This is definitely a limitation to this trial, in part because patients with stage I disease would undergo endoscopic treatment and those with stage IV disease may not undergo surgical resection. Third, histological structure differs greatly between CRC and $\mathrm{HCC} / \mathrm{RCC}$; invasive CRCs are relatively stroma-rich tumors whereas both HCCs and RCCs are deficient in fibrous stroma. In the present study, as well as in previous studies, $\alpha$-taxilin expression was also noted in the stromal cells such as fibroblasts. Previous studies suggested fibroblasts as the cellular origin of matrix metalloproteinase (MMP)-9 in CRCs $(20,21)$. Increased MMP-2 and MMP-9 expression has been associated with worse outcome of CRC (22-24). The fact that $\alpha$-taxilin preferentially binds to free syntaxins and thereby prevents formation of the SNARE complex suggests that $\alpha$-taxilin might act as a negative regulator of t-SNARE formation, leading to impaired intracellular vesicular transport $(8,9)$. SNAREs have been reported to be involved in secretion of MMP-2 and MMP-9 (25), therefore $\alpha$-taxilin expression in fibroblasts might cause decreased MMP-9 secretion, leading to a favorable prognosis of CRCs. $\alpha$-taxilin expression in both cancer cells and stromal cells may offset the prognostic effect in stroma-rich CRCs.

In conclusion, this is the first report of $\alpha$-taxilin expression in human colorectal tumors. In both adenoma and adenocarcinoma, $\alpha$-taxilin was significantly associated with cell proliferation activity. However, $\alpha$-taxilin expression levels were not significantly associated with local invasiveness or recurrence-free/overall survival of advanced CRCs. Taken together, our findings indicate that $\alpha$-taxilin is a cell proliferation marker in colorectal epithelial neoplasms, but cannot be a marker of malignancy, and its expression does not affect prognosis of CRC.

\section{Acknowledgements}

We are greatly indebted to Ms. Chiaki Matsuyama and Ms. Ayako Shimizu for their excellent technical assistance. This study was supported in part by JSPS KAKENHI (JP16K08695) from the Ministry of Education, Culture, Sports, Science and Technology of Japan.

\section{References}

1. World Cancer Research Fund/American Institute for Cancer Research: Food, Nutrition, Physical Activity and the Prevention of Cancer: A Global Perspective. AICR, Washington DC, pp280-288, 2007.

2. Torre LA, Bray F, Siegel RL, Ferlay J, Lortet-Tieulent J and Jemal A: Global cancer statistics, 2012. CA Cancer J Clin 65: 87-108, 2015.

3. Vogelstein B, Papadopoulos N, Velculescu VE, Zhou S, Diaz LA Jr and Kinzler KW: Cancer genome landscapes. Science 339: 1546-1558, 2013

4. Strömberg S, Agnarsdóttir M, Magnusson K, Rexhepaj E, Bolander A, Lundberg E, Asplund A, Ryan D, Rafferty M, Gallagher WM, et al: Selective expression of Syntaxin-7 protein in benign melanocytes and malignant melanoma. J Proteome Res 8: 1639-1646, 2009.

5. Hashimoto S, Onodera Y, Hashimoto A, Tanaka M, Hamaguchi M, Yamada A and Sabe H: Requirement for Arf6 in breast cancer invasive activities. Proc Natl Acad Sci USA 101: 6647-6652, 2004.

6. Bascom JL, Fata JE, Hirai Y, Sternlicht MD and Bissell MJ: Epimorphin overexpression in the mouse mammary gland promotes alveolar hyperplasia and mammary adenocarcinoma. Cancer Res 65: 8617-8621, 2005.

7. Chen YA and Scheller RH: SNARE-mediated membrane fusion. Nat Rev Mol Cell Biol 2: 98-106, 2001. 
8. Nogami S, Satoh S, Nakano M, Shimizu H, Fukushima H, Maruyama A, Terano A and Shirataki H: Taxilin; a novel syntaxin-binding protein that is involved in $\mathrm{Ca} 2+$-dependent exocytosis in neuroendocrine cells. Genes Cells 8: 17-28, 2003.

9. Nogami S, Satoh S, Nakano M, Terano A and Shirataki H: Interaction of taxilin with syntaxin which does not form the SNARE complex. Biochem Biophys Res Commun 311: 797-802, 2003.

10. Horii Y, Sakane H, Nogami S, Ohtomo N, Tomiya T and Shirataki H: Expression of $\alpha$-taxilin in the murine gastrointestinal tract: Potential implication in cell proliferation. Histochem Cell Biol 141: 165-180, 2014.

11. Sai XB, Makiyama T, Sakane H, Horii Y, Hiraishi H and Shirataki H: TSG101, a tumor susceptibility gene, bidirectionally modulates cell invasion through regulating MMP-9 mRNA expression. BMC Cancer 15: 933, 2015.

12. Oba-Shinjo SM, Bengtson MH, Winnischofer SM, Colin C, Vedoy CG, de Mendonça Z, Marie SK and Sogayar MC: Identification of novel differentially expressed genes in human astrocytomas by cDNA representational difference analysis. Brain Res Mol Brain Res 140: 25-33, 2005.

13. Ohtomo N, Tomiya T, Tanoue Y, Inoue Y, Nishikawa T, Ikeda H, Seyama Y, Kokudo N, Shibahara J, Fukayama M, et al: Expression of $\alpha$-taxilin in hepatocellular carcinoma correlates with growth activity and malignant potential of the tumor. Int J Oncol 37: 1417-1423, 2010.

14. Mashidori T, Shirataki H, Kamai T, Nakamura F and Yoshida K: Increased alpha-taxilin protein expression is associated with the metastatic and invasive potential of renal cell cancer. Biomed Res 32: 103-110, 2011

15. Hamilton SR, Bosman FT, Boffetta P, Ilyas M, Morreau H, Nakamura SI, Quirke P, Riboli E and Sobin LH: Carcinoma of the colon and rectum. In: WHO Classification of Tumours of the Digestive System. 4th edition. Bozman FT, Carneiro F, Hruban RH and Theise ND (eds): IARC Press, Lyon, pp134-146, 2010.

16. American Joint Committee on Cancer (AJCC): Colon and Rectum Cancer Staging. 7th edition. AJCC, Chicago, IL, 2009. $\mathrm{https} / / /$ cancerstaging.org/references-tools/quickreferences/ Documents/ColonMedium.pdf. Accessed May 25, 2017.

17. Imai Y: Poorly differentiated adenocarcinoma of the colon: Subsite location and clinicopathologic features. Int J Colorecta Dis 30: 187-196, 2015.
18. Sakakibara S, Nakadate K, Tanaka-Nakadate S, Yoshida K, Nogami S, Shirataki H and Ueda S: Developmental and spatial expression pattern of alpha-taxilin in the rat central nervous system. J Comp Neurol 511: 65-80, 2008.

19. Fodde R, Smits R and Clevers H: APC, signal transduction and genetic instability in colorectal cancer. Nat Rev Cancer 1: 55-67, 2001.

20. Roeb E, Dietrich CG, Winograd R, Arndt M, Breuer B, Fass J, Schumpelick V and Matern S: Activity and cellular origin of gelatinases in patients with colon and rectal carcinoma differential activity of matrix metalloproteinase-9. Cancer 92: 2680-2691, 2001

21. Behrens P, Mathiak M, Mangold E, Kirdorf S, Wellmann A, Fogt F, Rothe M, Florin A and Wernert N: Stromal expression of invasion-promoting, matrix-degrading proteases MMP-1 and -9 and the Ets 1 transcription factor in HNPCC carcinomas and sporadic colorectal cancers. Int J Cancer 107: 183-188, 2003.

22. Said AH, Raufman JP and Xie G: The role of matrix metalloproteinases in colorectal cancer. Cancers (Basel) 6: 366-375, 2014.

23. Zeng ZS, Huang Y, Cohen AM and Guillem JG: Prediction of colorectal cancer relapse and survival via tissue RNA levels of matrix metalloproteinase-9. J Clin Oncol 14: 3133-3140, 1996.

24. Zeng ZS, Cohen AM and Guillem JG: Loss of basement membrane type IV collagen is associated with increased expression of metalloproteinases 2 and 9 (MMP-2 and MMP-9) during human colorectal tumorigenesis. Carcinogenesis 20: 749-755, 1999.

25. Kean MJ, Williams KC, Skalski M, Myers D, Burtnik A, Foster D and Coppolino MG: VAMP3, syntaxin-13 and SNAP23 are involved in secretion of matrix metalloproteinases, degradation of the extracellular matrix and cell invasion. J Cell Sci 122: 4089-4098, 2009. 